\title{
Control-Informed Optimal Array Layout for Wave Farms
}

\author{
Paula B. Garcia-Rosa, Member, IEEE, Giorgio Bacelli, and John V. Ringwood, Senior Member, IEEE
}

\begin{abstract}
Somewhat similar to wind farms, commercial wave energy will be realized using farms of devices. However, for wave farms, both constructive and destructive interference may occur between individual devices; whereas, for wind farms, only destructive interference occurs. In order to maximize constructive interference, the layout of the wave farm must be optimized. In addition, power absorption may be improved by applying a control system technology to the farm of devices. As with many applications, there is a tendency in wave energy to separate the design of the array layout from the design of an energy maximizing control system. In this paper, we show that integrating the activities of optimal array layout design and control design are crucial if the full potential of the wave farm is to be realized. In particular, we show that a layout optimized without knowledge of the control system to be used can be inferior to the extent of recovering $40 \%$ less energy than a farm layout optimized with knowledge of the control system. This loss is significantly more than the difference in performance between a simple and a very complex control strategy.
\end{abstract}

Index Terms-Control of arrays, optimal array layout, power optimization, wave energy farm.

\section{INTRODUCTION}

$\mathbf{T}$ HE COMMERCIAL operation of most designed wave energy converters (WECs) relies on the deployment of wave farms. On a wave farm, multiple units are connected together in an array to provide a significant contribution of electricity to power grids and also to provide some power smoothing. Therefore, each single converter is subject to waves that have been radiated or diffracted from other converters, in addition to external (incident) waves. The effect of array interactions on the energy absorption may be either constructive or destructive, depending on whether the averaged energy of each unit in the array is higher or lower than the energy of isolated units. Such an effect is usually quantified by the $q$ factor [1].

The $q$ factor is defined as [1]

$$
q=\frac{W}{n W_{s}}
$$

Manuscript received April 22, 2014; revised September 06, 2014 and November 14, 2014; accepted December 20, 2014. Date of publication February 27, 2015; date of current version March 18, 2015. This work was supported by the Science Foundation Ireland under Grant 12/RC/2302 for the Marine Renewable Ireland (MaREI) Centre. This work was completed while G. Bacelli was affiliated with the Centre for Ocean Energy Research at Maynooth University. Paper no. TSTE-00171-2014.

P. B. Garcia-Rosa and J. V. Ringwood are with the Centre for Ocean Energy Research, Maynooth University, County Kildare, Ireland (e-mail: paula.garciarosa@eeng.nuim.ie; john.ringwood@eeng.nuim.ie).

G. Bacelli was with the Centre for Ocean Energy Research, Maynooth University, County Kildare, Ireland. $\mathrm{He}$ is now with the Water Power Technologies Department, Sandia National Laboratories, Albuquerque, NM 87123 USA (e-mail: gbacelli@ sandia.gov).

Color versions of one or more of the figures in this paper are available online at http://ieeexplore.ieee.org.

Digital Object Identifier 10.1109/TSTE.2015.2394750 where $W$ is the total energy absorbed by the array, $W_{s}$ is the energy absorbed for a single isolated device, and $n$ is the number of units in the array. If wave interactions have a constructive effect on the absorbed energy, then $q>1$ and, if the effect is destructive, $q<1$.

Many research studies on wave energy farms have been performed over the last few decades and the development of optimal array layouts still remains a challenging problem. Overall, the optimal layout is a function of the hydrodynamic interactions (amount of power), cost of moorings [2] and electrical interconnections [3], smoothing properties (quality of power) and maintainable costs. In this paper, we focus on the optimal hydrodynamical array layout.

Focusing primarily on closely spaced arrays and point absorber devices, pioneering studies on wave farms have shown that the array performance (or the $q$ factor) depends on the frequencies of the incident waves and on the array layout [1], [4], [5]. Since these pioneering studies, some theoretical research on aspects of the hydrodynamic characteristics of wave farms have been presented, e.g., [6]-[9].

Recent research on optimal hydrodynamical array layouts has studied the influence of different factors on array performance. For example, factors such as configuration, separating distance, number of units, and wave direction are analyzed for different wave climates in [10]. However, given that devices in a wave farm can experience both destructive and constructive interference, separating distance has been shown to be a key factor in obtaining the highest $q$ factor values [10]. In [11], Engström et al. showed that the variance of the power production for rectangular arrays is, on average, three times higher than the variance of the power for circular arrays. The spatial configuration is also investigated in [12], where two different methods based on genetic algorithms and parabolic intersection methods have been proposed to optimize an array layout with five devices. In addition, it has been shown that the average of the $q$ factor over incident wave directions from 0 to $2 \pi$ is always equal to unity [13]. This means that arrays with high values of $q$ at some wave directions are associated with unfavourable values at some other directions.

A number of studies have investigated the influence of control strategies on wave energy array performance. Focusing on suboptimal strategies based on linear damping [14], [15], it has been shown that the increase in the average interaction factor for irregular waves (IW) may be less than that regular wave (RW) analysis suggests, but it is not insignificant. Different strategies based on model-predictive control [16], [17] have shown that higher performance improvements can be achieved when the controllers of each unit run cooperatively by 
transmitting information to each other. However, the focus of all of these array control studies is to design the control to maximize the power capture for a given array layout. This approach follows the standard sequential design procedure, where application systems are designed by application specialists and the control system is subsequently added. In this study, we consider simultaneous design of the array layout and control system.

By virtue of conservation of energy, a WEC must interact with the waves such as to reduce the amount of wave energy that is otherwise present in the sea. The device must be able to generate waves, which interfere destructively with the sea waves [18]. Thus, in order to absorb power, a certain fraction of the excitation power arriving at the device is necessarily returned to the sea as radiated power. The radiated wave interferes with the incident wave and the resulting wave, which propagates beyond the device, transports less power than the incident wave [18].

Control strategies for optimizing the absorbed energy modify the oscillation of each device and consequently, the radiated wave patterns are also modified. For RW and optimum oscillation conditions of the body, the radiated power equals the absorbed power [19]. Therefore, it is expected that the optimal hydrodynamical array layout will be different, depending on whether the WEC is controlled or not and how it is controlled. In particular, some (global) control strategies can take array interactions into account [16] while other (independent) strategies do not. In this paper, we show that highly suboptimal array layouts result from optimizing the array layout for one particular controller (or no controller) and subsequently employing a different one. To illustrate the impact of such effects, the influence of three different control strategies on the optimal array layout is evaluated. The control strategies employed are: passive control (PC), global control (GC), and independent control (IC). GC and IC algorithms considered in this study were proposed in [16]. Since these strategies are model-based control strategies, the formulation of the hydrodynamic model presented here follows the hydrodynamic model formulation of [16]. A preliminary study examining the effect of control strategies on optimal wave farm layout was presented in [20], which compared global array control to passive damping, for a fixed incident wave angle.

\section{EQUATIONS OF MOTION}

Linear hydrodynamic theory and heave oscillatory motion are assumed. In such a case, the motion of the floating bodies can be described by the Cummins equation [21]

$$
\mathbf{M}_{t} \ddot{\mathbf{x}}(t)+\mathbf{B}_{v} \dot{\mathbf{x}}(t)+\int_{0}^{\infty} \mathbf{K}(t-\tau) \dot{\mathbf{x}}(\tau) d \tau+\mathbf{S}_{h} \mathbf{x}(t)=\mathbf{f}(t)
$$

with $\mathbf{M}_{t}=\left[\mathbf{M}+\mathbf{M}_{r}(\infty)\right], \mathbf{f}(t)=\mathbf{f}_{e}(t)+\mathbf{f}_{p}(t)$, and the kernel of the convolution term (fluid memory term) expressed as

$$
\mathbf{K}(t-\tau)=\frac{2}{\pi} \int_{0}^{\infty} \mathbf{B}_{r}(\omega) \cos [\omega(t-\tau)] d \omega
$$

where

$$
\begin{array}{ll}
\mathbf{x}(t) \in \mathrm{R}^{n} & \text { is the vector of vertical positions of the } n \\
& \text { bodies; } \\
& \text { is the mass matrix of the system and } \\
& \mathbf{M}_{r}(\infty) \in \mathrm{R}^{n \times n}(\infty n \text { is the added mass } \\
& \text { matrix, defined with the asymptotic } \\
& \text { values of the added masses at infinite } \\
& \text { frequency; } \\
& \text { is the hydrodynamic stiffness matrix; } \\
& \text { is the viscous damping matrix, } \\
& \mathbf{B}_{r}(\omega) \in \mathrm{R}^{n \times n} \text { is the radiation resistance } \\
& \text { matrix, and } \omega \text { is the wave frequency; } \\
\mathbf{S}_{h} \in \mathrm{R}^{n \times n} \in \mathrm{R}^{n \times n} & \text { is the vector of excitation forces. The } \\
& \text { excitation force is calculated as } \\
& \mathbf{f}_{e}(t)=\mathcal{F}-1\left\{\mathbf{F}_{e}(\omega) \eta(\omega)\right\}, \text { where } \eta(\omega) \\
& \text { is the Fourier transform of the wave } \\
\mathbf{f}_{e}(t) & \text { elevation and } \mathbf{F}_{e}(\omega) \text { is the excitation } \\
& \text { force transfer function. The excitation } \\
& \text { power is the energy delivered by the } \\
& \text { incoming waves to each device, i.e., } \\
& \mathbf{P}_{e}(t)=\mathbf{f}_{e}(t) \dot{\mathbf{x}}(t) \text { [19]; } \\
& \text { is the vector of power take-off (PTO) } \\
& \text { forces. } \boldsymbol{f}_{p}(\mathrm{t}) \text { is described in the next } \\
& \text { section according to the control strategy } \\
& \text { applied to the } \mathbf{W E C .}
\end{array}
$$

Matrices $\mathbf{F}_{e}(\omega), \mathbf{B}_{r}(\omega), \mathbf{M}_{r}(\infty)$, and $\mathbf{S}_{h}$ are computed using the boundary element solver WAMIT [22]. The viscous damping matrix $\mathbf{B}_{v}$ is given by $\mathbf{B}_{v}=B_{v} \mathbf{I}_{n}$, where $\mathbf{I}_{n}$ is the identity matrix of order $n$. From Lorentz linearization [23], the coefficient $B_{v}$ is calculated for an isolated device as

$$
B_{v}=\frac{\rho C_{d} A}{2} \frac{\int_{0}^{T} \dot{x}^{2}(t)|\dot{x}(t)| d t}{\int_{0}^{T} \dot{x}^{2}(t) d t}
$$

where $\rho$ is the fluid density, $C_{d}$ is the drag coefficient, $A$ is the area of the body projected onto the plane orthogonal to the velocity $\dot{x}$, and $T$ is the time interval.

In order to more easily formulate the control problem, the system equations are discretized by approximating the velocity and the PTO force with a linear combination of basis functions. Following the procedure from [16], trigonometric functions are chosen as basis functions. Then, the PTO force and the velocity are, respectively, approximated with the truncated zero-mean Fourier series

$$
\begin{aligned}
\dot{x}_{k}(t) & \approx \sum_{n_{f}=1}^{N / 2} \nu_{k}^{n_{f}, c} \cos \left(n_{f} \omega_{0} t\right)+\nu_{k}^{n_{f}, s} \sin \left(n_{f} \omega_{0} t\right) \\
f_{p, k}(t) & \approx \sum_{n_{f}=1}^{N / 2} \phi_{k}^{n_{f}, c} \cos \left(n_{f} \omega_{0} t\right)+\phi_{k}^{n_{f}, s} \sin \left(n_{f} \omega_{0} t\right)
\end{aligned}
$$

where $\omega_{0}$ is the fundamental frequency of the Fourier series, $\nu_{k}$ and $\phi_{k}$ are, respectively, the Fourier coefficients of the velocity and the PTO force of the $k$ th device, and $N$ is the total number of components. 
The best approximation of the solution for the equation of motion (2) is sought by applying the Galerkin method (see [24] for more details), and the result is the linear system

$$
\mathcal{G} \mathcal{X}=\mathcal{P}+\mathcal{E}
$$

where $\mathcal{X}$ represents the vector of velocities, $\mathcal{P}$ represents the vector of PTO forces, $\mathcal{E}$ is the vector of excitation forces and $\mathcal{G}$ is the matrix of hydrodynamical coefficients of the system. $\mathcal{X}$, $\mathcal{P}, \mathcal{E}$, and $\mathcal{G}$ are, respectively, defined for $n$ units as

$$
\begin{aligned}
\mathcal{X} & =\left[\begin{array}{c}
X_{1} \\
X_{2} \\
\vdots \\
X_{n}
\end{array}\right] \mathcal{P}=\left[\begin{array}{c}
P_{1} \\
P_{2} \\
\vdots \\
P_{n}
\end{array}\right] \mathcal{E}=\left[\begin{array}{c}
\mathrm{E}_{1} \\
\mathrm{E}_{2} \\
\vdots \\
\mathrm{E}_{n}
\end{array}\right] \\
\mathcal{G}= & {\left[\begin{array}{cccc}
\mathrm{G}_{11} & \mathrm{G}_{12} & \ldots & \mathrm{G}_{1 n} \\
\mathrm{G}_{21} & \mathrm{G}_{22} & \ldots & \mathrm{G}_{2 n} \\
\vdots & \vdots & \ddots & \vdots \\
\mathrm{G}_{n 1} & \mathrm{G}_{n 2} & \ldots & \mathrm{G}_{n n}
\end{array}\right] . }
\end{aligned}
$$

The vectors $X_{k}$ and $P_{k}$ are the vectors of the Fourier coefficients of the velocity and PTO force of the $k$ th device, respectively. The vectors are arranged as

$$
\begin{aligned}
& X_{k}=\left[\nu_{k}^{1, c}, \nu_{k}^{1, s}, \nu_{k}^{2, c}, \nu_{k}^{2, s}, \ldots, \nu_{k}^{\frac{N}{2}, c}, \nu_{k}^{\frac{N}{2}, s}\right]^{T} \\
& P_{k}=\left[\phi_{k}^{1, c}, \phi_{k}^{1, s}, \phi_{k}^{2, c}, \phi_{k}^{2, s}, \ldots, \phi_{k}^{\frac{N}{2}, c}, \phi_{k}^{\frac{N}{2}, s}\right]^{T} .
\end{aligned}
$$

The elements of the vectors $E_{k}$ are the Fourier coefficients of the excitation force on the $k$ th device and are arranged in the same manner as the vectors $X_{k}$ and $P_{k}$. The matrices $\mathrm{G}_{i k} \in$ $\mathrm{R}^{N \times N}$ composing the matrix $\mathcal{G}$ are block diagonal, where each of the $N / 2$ square blocks is of size two and the $l$-th block is defined as

$$
\begin{aligned}
\mathrm{G}_{i k}^{l} & =\left[\begin{array}{cc}
\mathrm{D}_{i k}^{l} & \mathrm{C}_{i k}^{l} \\
-\mathrm{C}_{i k}^{l} & \mathrm{D}_{i k}^{l}
\end{array}\right] \quad \text { for } l=1, \ldots, N / 2 \\
\mathrm{D}_{i k}^{l} & =\mathrm{B}_{r, i k}\left(l \omega_{0}\right)+\mathrm{B}_{v, i k} \\
\mathrm{C}_{i k}^{l} & =l \omega_{0}\left(\mathrm{M}_{i k}+\mathrm{M}_{r, i k}\left(l \omega_{0}\right)\right)-\mathrm{S}_{h, i k} /\left(l \omega_{0}\right) .
\end{aligned}
$$

$\mathrm{B}_{v, i k}, \mathrm{M}_{i k}, \mathrm{~S}_{h, i k}$ and $\mathrm{B}_{r, i k}(\omega)$ are, respectively, the elements of the matrices $\mathbf{B}_{v}, \mathbf{M}, \mathbf{S}_{h}$, and $\mathbf{B}_{r}(\omega) . \mathbf{M}_{r, i k}(\omega)$ are the elements of the added mass matrix $\mathbf{M}_{r}(\omega)$, which is also computed by the solver WAMIT.

\section{OptimizATION OF THE ARRAY LAYOUT}

\section{A. Optimization Problem}

The optimal hydrodynamic design of wave farms should maximize constructive interference between the units [6], or maximize the $q$ factor. The $q$ factor (1) is a dimensionless number, which represents the average energy of each unit in the array normalized by the energy captured by a single device. A $q$ factor of unity indicates that, on average, each device in the array is capturing the same power as an isolated device.
The total energy absorbed by the array, over a time interval $T$, is the sum of the energy absorbed by each body [16]

$$
W=-\sum_{k=1}^{n} \int_{0}^{T} \dot{x}_{k}(t) f_{p, k}(t) d t
$$

where $f_{p, k}$ is the PTO force (determined by the control strategy) applied to each floating body $k$. The energy absorbed by a single device $\left(W_{s}\right)$ is a particular case of (10) and (2) with $n=1$. Note that $W_{s}$ is calculated following the same scenario considered for the arrays, i.e., same incident waves and control algorithm employed. Following the notation of (7), the total energy absorbed (10) is rewritten [16] as

$$
\mathcal{W}=-\mathcal{P}^{T} \mathcal{X}
$$

where $\mathcal{X}$ and $\mathcal{P}$ are, respectively, the vectors of the Fourier coefficients of the velocity and PTO force.

This work studies the optimal distance between the units in a wave farm and the optimal array orientation, for a given array configuration and different control strategies. Thus, the optimal array layout problem is defined as: Determine the optimal parameters (separating distancelarray orientation) that maximize the q factor (1), for different controllers. The PTO force calculations are described next for PC, GC, and IC.

\section{B. Passive Control}

A generic PTO system with a linear damper $\left(B_{p} \in \mathrm{R}_{+}\right)$is considered. The PTO forces are given in the time domain by

$$
f_{p, k}(t)=-B_{p} \dot{x}_{k}(t) .
$$

From (7), the Fourier coefficients of the PTO forces are

$$
\mathcal{P}=-\mathcal{B} \mathcal{X}
$$

where $\mathcal{B}=B_{p} \mathbf{I}_{k N}$ and $\mathbf{I}_{k N}$ is the identity matrix of order $k N$. For $\mathrm{PC}$, the coefficient $B_{p}$ is given by

$$
B_{p}=\sqrt{\left(B_{r}(\omega)\right)^{2}+\left(\omega\left(M+M_{r}(\omega)\right)-S_{h} / \omega\right)^{2}}
$$

where $\omega$ is the frequency of the incident wave. In this case, $M, B_{r}, M_{r}$, and $S_{h}$ are the mass, radiation damping, added mass and hydrostatic stiffness coefficients of an isolated device, respectively. Equation (14) represents optimal linear damping when the bodies are subjected to incident RW [19]. IW are not defined by a single frequency in the time-domain. For this reason, a frequency that best characterizes the wave spectrum should be selected. Here, $B_{p}$ is tuned to the peak frequency of the spectrum $\omega_{p}$ for each sea state.

\section{Global Control}

The GC strategy is aware of the full array system. In this case, the PTO forces are obtained by solving the optimal energy absorption problem, defined as: Maximize the performance function (11) subject to (7). We assume that the wave excitation is known completely into the future, or can be forecast, and $\mathcal{G}$ is nonsingular. Then, the optimal force coefficients $\mathcal{P}^{*}$, which 
maximize the array absorbed energy, are obtained by solving the optimization problem [16]

$$
\mathcal{P}^{*}=\arg \max _{\mathcal{P}}\left(-\mathcal{P}^{T} \mathcal{G}^{-1} \mathcal{P}-\mathcal{P}^{T} \mathcal{G}^{-1} \mathcal{E}\right)
$$

where $\mathcal{P}, \mathcal{G}$, and $\mathcal{E}$ are defined in (7).

\section{Independent Control}

IC assumes that each device is equipped with its own individual controller and excitation force estimator [16]. Each controller uses the model of a single isolated unit, having no communication with other devices in the array or any knowledge of them. Therefore, the optimal PTO force coefficients are obtained by solving each individual optimization problem for the $k$ th body

$$
P_{k}^{*}=\arg \max _{P_{k}}\left(-P_{k}^{T} G_{s}^{-1} P_{k}-P_{k}^{T} G_{s}^{-1} \bar{E}_{k}\right)
$$

with cost functions

$$
W_{k}=-P_{k}^{T} X_{k}
$$

where $\mathrm{G}_{s} \in \mathrm{R}^{N \times N}$ is analogous to the matrix $\mathcal{G}$ in (7), but now calculated using the hydrodynamic coefficients of a single isolated device, and $\overline{\mathrm{E}}_{k}$ is the excitation force measured by the estimator on body $k$. It is assumed that the excitation force estimator on each device cannot discern the excitation force due to incoming waves from the radiation generated by other bodies (for more details see [16]).

\section{Sensitivity of the Optimal Array Layout TO DIFFERENT CONTROL STRATEGIES}

This section examines the sensitivity of the optimal array layout to the following control strategies: PC, GC, and IC. The aim is to investigate how the controllers affect the optimal distance $d$ between the units, and the optimal array orientation $\beta$. In particular, we aim to show that the optimal array layout is highly dependent on the control strategy employed.

Simulation with RW and IW is performed. The RW are defined by wave height $H$ and period $T_{w}$. The IW are calculated as a linear combination of RW using a Bretschneider spectrum envelope, defined by significant wave height $H_{s}$, and peak period $T_{p}$. The optimal layout parameters are determined via simulations with a complete set of possible values of $d / r$ and $\beta$, where $r$ is the device radius. As a result, a clear overview of the mapping between the $q$ factor and the optimization parameters $(d / r$ and $\beta)$ can be observed.

\section{A. Candidate Array Layouts}

Fig. 1 illustrates the array layouts considered, where $\beta$ is the incident wave direction. Layout $1(L 1)$ has two floating bodies, Layout $2(L 2)$ is a linear array of three bodies, Layout $3(L 3)$ is composed of three bodies placed at the vertices of an equilateral triangle, and Layout $4(L 4)$ is a square array of four floating bodies. All arrays are composed of heaving cylinder devices, with a number of different device geometries considered. Each geometry has approximately the same volume $\left(\approx 160 \pi \mathrm{m}^{3}\right)$, but different radius $r$, draught $h$, and resonance period $T_{r}$, as described in Table I.
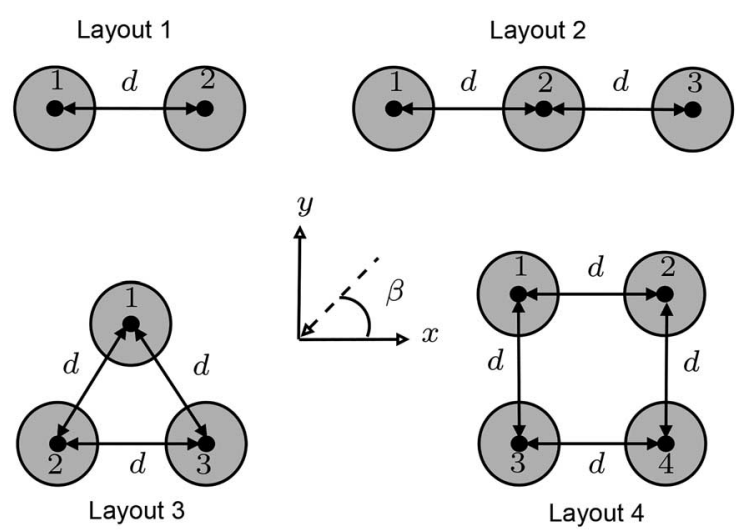

Fig. 1. Top view of array layouts and incident wave direction $\beta$.

TABLE I

SET OF CYLINDERS CONSIDERED

\begin{tabular}{|c|c|c|c|}
\hline WEC Geometry & G1 & G2 & G3 \\
\hline Radius $r(\mathrm{~m})$ & 4 & 6.25 & 8 \\
Draught $h(\mathrm{~m})$ & 10 & 4 & 2.5 \\
Resonance $T_{r}(\mathrm{~s})$ & 7.1 & 5.4 & 5 \\
\hline
\end{tabular}

\section{B. Influence of the Distance}

Here, the aim is to investigate how the optimal values of the separating distance change, as different control strategies are considered. For each simulation, the direction of propagation of the waves $\beta$ is constant. In order to avoid shadowing effects, a different value of $\beta$ is considered depending on the configuration of the arrays: $\beta=90^{\circ}$ for linear configurations $(L 1-L 2)$, $\beta=0^{\circ}$ for $L 3$, and $\beta=45^{\circ}$ for $L 4$.

Initially, we consider the simplest array layout $L 1$, geometry $G 2$, and RW ( $H=1 \mathrm{~m} ; T_{w}=9 \mathrm{~s}$ ). Fig. 2 shows the evolution of the $q$ factor and the total energy absorbed $W$ versus $d / r$ for $\beta=90^{\circ}$. Different optimal $d / r$ values are obtained for different control strategies, especially between GC and PC strategies. From Fig. 2, it is clear that maximizing the $q$ factor also maximizes the total absorbed energy for a certain incident wave regime and control strategy. Although GC and IC show lower $q$ factor values than $\mathrm{PC}$ at some $d / r$ ratios [Fig. 2(a)], the active controllers result in higher captured energy levels than the passive controller [Fig. 2(b)].

Table II summarizes the simulation results for the simplest array layout, considering the three geometries in Table I. It can be noted that the optimal values of $d / r$ are larger for geometries with strong radiation properties (e.g., $G 1$, which has a large $d / r$ ratio) and higher when the periods of the incident wave are longer. Comparing the results for GC and PC, larger distances are required on GC for optimal configuration, but when $T_{w} \rightarrow$ $T_{r}$ the optimal distances are the same. In this case, the devices are at resonance with the waves and the control strategies exert no effort.

Fig. 3 shows the evolution of the $q$ factor versus $d / r$ for $\beta=90^{\circ}$ and IW $\left(H_{s}=1 \mathrm{~m} ; T_{p}=9 \mathrm{~s}\right)$. Note that the $q$ factor is more sensitive to GC (and IC) than PC. Actually, both GC and IC actively modify the dynamics of each device, resulting in more exaggerated motion of the bodies and more absorbed energy than PC, as illustrated in Fig. 2(b). As a result, the active controllers cause more constructive or destructive effects than 


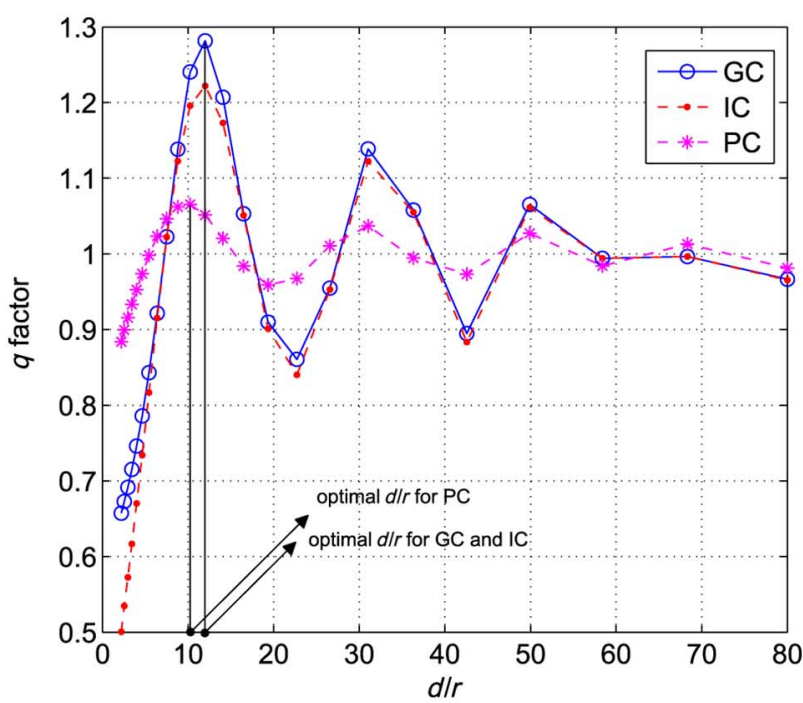

(a)

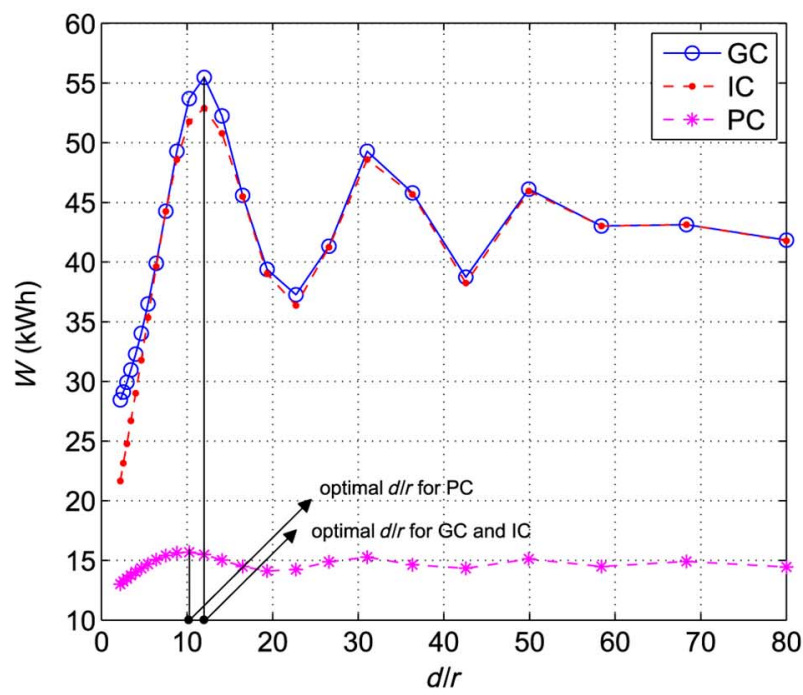

(b)

Fig. 2. $L 1, G 2$ : (a) evolution of the $q$ factor and (b) evolution of the total absorbed energy by the array versus $d / r$ for constant $\beta=90^{\circ}$ (RW: $H=1 \mathrm{~m}$; $\left.T_{w}=9 \mathrm{~s}\right)$.

TABLE II

$L 1$-Optimal Values of $d / r\left(\mathrm{RW}: H=1 \mathrm{M}\right.$ AND $\left.\beta=90^{\circ}\right)$

\begin{tabular}{|c|c|c|c|c|c|c|}
\hline \multirow{2}{*}{ Geometry } & \multicolumn{3}{|c|}{$T_{w}=5 \mathrm{~s}$} & \multicolumn{3}{c|}{$T_{w}=7 \mathrm{~s}$} \\
\cline { 2 - 7 } & IC & GC & PC & IC & GC & PC \\
\hline G1 & 4 & 4 & 4 & 10 & 10 & 10 \\
G2 & 3.5 & 3.5 & 3.5 & 6.4 & 6.4 & 5.4 \\
G3 & 3 & 3 & 3 & 5.4 & 5.4 & 4.6 \\
\hline \hline \multirow{2}{*}{ Geometry } & \multicolumn{3}{|c|}{$T_{w}=9 \mathrm{~s}$} & \multicolumn{3}{c|}{$T_{w}=11 \mathrm{~s}$} \\
\cline { 2 - 7 } & IC & GC & PC & IC & GC & PC \\
\hline G1 & 17 & 17 & 17 & 29.3 & 29.3 & 24.5 \\
G2 & 12 & 12 & 10.2 & 16.5 & 16.5 & 14.1 \\
G3 & 9.5 & 9.5 & 8.3 & 12.8 & 12.8 & 11 \\
\hline
\end{tabular}

PC, when different $d / r$ ratios are considered. In addition, the evolution of the $q$ factor for the IC strategy is not significantly different from GC (same optimal values for $d / r$ ) and is omitted from the following analysis.

Table III summarizes the optimal $d / r$ values for the array layout $L 1$ considering the three geometries and different

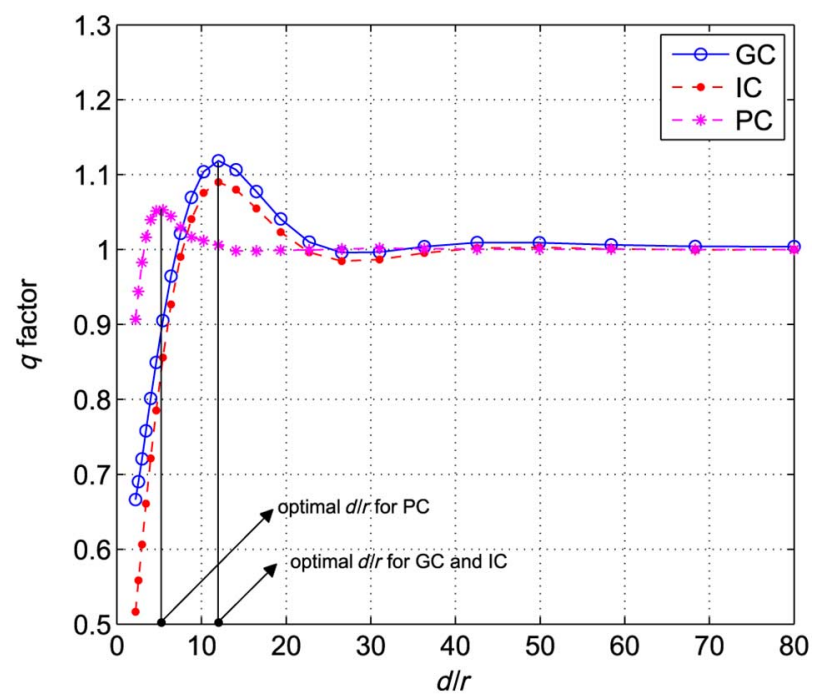

Fig. 3. $L 1, G 2$ : evolution of the $q$ factor versus $d / r$ for constant $\beta=90^{\circ}$ (IW: $H_{s}=1 \mathrm{~m} ; T_{p}=9 \mathrm{~s}$ ).

TABLE III

$L 1$-Optimal Values of $d / r\left(\mathrm{IW}: H_{s}=1 \mathrm{M}\right.$ AND $\left.\beta=90^{\circ}\right)$

\begin{tabular}{|c|c|c|c|c|c|c|c|c|}
\hline \multirow{2}{*}{ Geometry } & \multicolumn{2}{|c|}{$T_{p}=5 \mathrm{~s}$} & \multicolumn{2}{c|}{$T_{p}=7 \mathrm{~s}$} & \multicolumn{2}{c|}{$T_{p}=9 \mathrm{~s}$} & \multicolumn{2}{c|}{$T_{p}=11 \mathrm{~s}$} \\
\cline { 2 - 9 } & $\mathrm{GC}$ & $\mathrm{PC}$ & $\mathrm{GC}$ & $\mathrm{PC}$ & $\mathrm{GC}$ & $\mathrm{PC}$ & $\mathrm{GC}$ & $\mathrm{PC}$ \\
\hline $\mathrm{G} 1$ & 7 & 4.8 & 11.8 & 8.3 & 20.5 & 10 & 24.5 & 10 \\
$\mathrm{G} 2$ & 4.6 & 3.5 & 7.5 & 4 & 12 & 5.4 & 16.5 & 6.4 \\
$\mathrm{G} 3$ & 4 & 3 & 6.1 & 3.4 & 9.5 & 4.6 & 14.7 & 5.4 \\
\hline
\end{tabular}

TABLE IV

$L 2$-Optimal Values of $d / r\left(\mathrm{IW}: H_{s}=1 \mathrm{M}\right.$ AND $\beta=90^{\circ}$ )

\begin{tabular}{|c|c|c|c|c|c|c|c|c|}
\hline \multirow{2}{*}{ Geometry } & \multicolumn{2}{|c|}{$T_{p}=5 \mathrm{~s}$} & \multicolumn{2}{c|}{$T_{p}=7 \mathrm{~s}$} & \multicolumn{2}{c|}{$T_{p}=9 \mathrm{~s}$} & \multicolumn{2}{c|}{$T_{p}=11 \mathrm{~s}$} \\
\cline { 2 - 9 } & $\mathrm{GC}$ & $\mathrm{PC}$ & $\mathrm{GC}$ & $\mathrm{PC}$ & $\mathrm{GC}$ & $\mathrm{PC}$ & $\mathrm{GC}$ & $\mathrm{PC}$ \\
\hline G1 & 7 & 4 & 11.8 & 7 & 17 & 8.3 & 24.5 & 10 \\
$\mathrm{G} 2$ & 4.6 & 3.5 & 6.4 & 4.6 & 12 & 4.6 & 16.5 & 6.4 \\
$\mathrm{G} 3$ & 4 & 3.5 & 5.4 & 4 & 9.5 & 4 & 12.75 & 5.4 \\
\hline
\end{tabular}

TABLE V

$L 3$-Optimal Values of $d / r\left(\mathrm{IW}: H_{s}=1 \mathrm{M}\right.$ AND $\beta=0^{\circ}$ )

\begin{tabular}{|c|c|c|c|c|c|c|c|c|}
\hline \multirow{2}{*}{ Geometry } & \multicolumn{2}{|c|}{$T_{p}=5 \mathrm{~s}$} & \multicolumn{2}{c|}{$T_{p}=7 \mathrm{~s}$} & \multicolumn{2}{c|}{$T_{p}=9 \mathrm{~s}$} & \multicolumn{2}{c|}{$T_{p}=11 \mathrm{~s}$} \\
\cline { 2 - 9 } & $\mathrm{GC}$ & $\mathrm{PC}$ & $\mathrm{GC}$ & $\mathrm{PC}$ & $\mathrm{GC}$ & $\mathrm{PC}$ & $\mathrm{GC}$ & $\mathrm{PC}$ \\
\hline G1 & 17 & 3.4 & 29.3 & 4.8 & 42.3 & 4.8 & 60.5 & 5.8 \\
G2 & 12 & 7.5 & 16.5 & 12 & 26.6 & 12 & 36.3 & 22.7 \\
$\mathrm{G} 3$ & 7.1 & 6.1 & 12.8 & 9.5 & 22.8 & 9.5 & 30.4 & 17 \\
\hline
\end{tabular}

TABLE VI

$L 4$-Optimal Values of $d / r\left(\mathrm{IW}: H_{s}=1 \mathrm{M}\right.$ AND $\beta=45^{\circ}$ )

\begin{tabular}{|c|c|c|c|c|c|c|c|c|}
\hline \multirow{2}{*}{ Geometry } & \multicolumn{2}{|c|}{$T_{p}=5 \mathrm{~s}$} & \multicolumn{2}{c|}{$T_{p}=7 \mathrm{~s}$} & \multicolumn{2}{c|}{$T_{p}=9 \mathrm{~s}$} & \multicolumn{2}{c|}{$T_{p}=11 \mathrm{~s}$} \\
\cline { 2 - 9 } & $\mathrm{GC}$ & $\mathrm{PC}$ & $\mathrm{GC}$ & $\mathrm{PC}$ & $\mathrm{GC}$ & $\mathrm{PC}$ & $\mathrm{GC}$ & $\mathrm{PC}$ \\
\hline G1 & 29.3 & 3 & 42.3 & 4 & 72.5 & 4.8 & 87 & 4.8 \\
G2 & 19.6 & 16.5 & 26.6 & 22.7 & 42.6 & 22.7 & 58.4 & 31 \\
G3 & 14.8 & 11 & 19.6 & 14.8 & 30.4 & 17 & 46.8 & 22.8 \\
\hline
\end{tabular}

periods of incident IW. The relative difference between the optimal $d / r$ values for GC and PC is greater for longer periods and smaller when $T_{p} \rightarrow T_{r}$, as the control strategies do not exert much effort in such a case.

Tables IV-VI summarize the optimal $d / r$ values for GC and PC, respectively, for the array layouts $L 2-L 4$, also for the IW case. The results in Table IV for $L 2$ are similar to those obtained for $L 1$, since both are linear arrays. However, the optimal $d / r$ values are greater as the number of WECs increases and the 


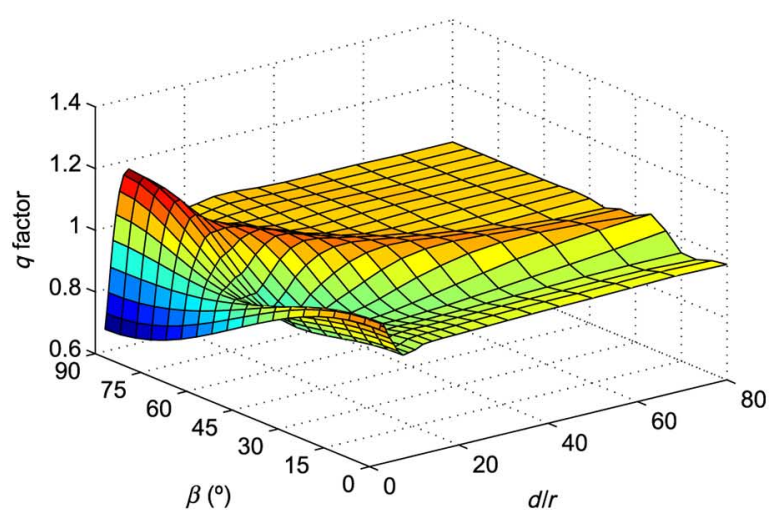

(a)

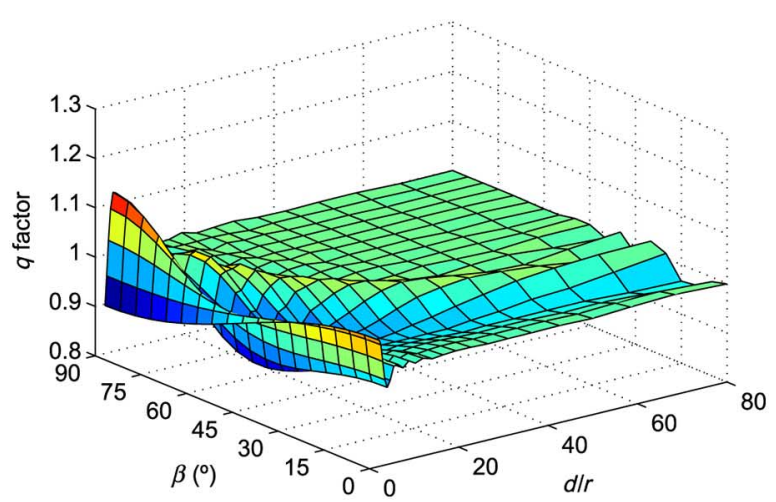

(b)

Fig. 4. $L 1, G 2$ : evolution of the $q$ factor for different values of $d / r$ and $\beta$. (a) GC. (b) PC (IW: $H_{s}=1 \mathrm{~m} ; T_{p}=7 \mathrm{~s}$ ).

interactions become more complex. Exceptions occur when PC is applied to the triangular and square layouts, for geometry $G 1$.

\section{Influence of Array Orientation}

Figs. 4-7 illustrate the evolution of the $q$ factor for different values of $d / r$ ratios and angles $\beta$, layouts $L 1-L 4$ and geometry $G 2$, when the WECs are subject to IW with $H_{s}=1 \mathrm{~m}$ and $T_{p}=7 \mathrm{~s}$. In this case, $T_{p}>T_{r}$ and the effect of different control strategies on the optimal layout can be better observed.

For linear arrays, the optimal $\beta$ is $90^{\circ}$ for both GC and PC strategies, as can be seen in Figs. 4 and 5. For symmetry reasons, the wave direction affects the interactions in the same way for both bodies, as shown in [25]. The influence of the incident wave direction on the linear arrays are almost the same for both strategies, except that GC shows a higher $q$ factor sensitivity, as explained previously in Section IV-B. For the triangular array ( $L 3)$, the optimal $\beta$ values for both control strategies are achieved for $0^{\circ}, 30^{\circ}, 60^{\circ}$, or $90^{\circ}$, when the wave direction is aligned with one side of the triangle. In addition, the optimal $\beta$ is $45^{\circ}$ for the square array $(L 4)$.

Although the optimal array orientations for the studied configurations are the same for both GC and PC strategies, the evolution of the $q$ factor is different for each strategy, as is illustrated in Figs. 6 and 7. The GC curve for array L4, e.g., shows a maximum peak at $\beta=45^{\circ}, d / r \approx 27$ [Fig. 7(a)]. Nevertheless, the PC curve [Fig. 7(b)] shows a maximum peak at $\beta=45^{\circ}$, $d / r \approx 23$ and a minimum peak at $\beta=45^{\circ}, d / r \approx 10$. This can

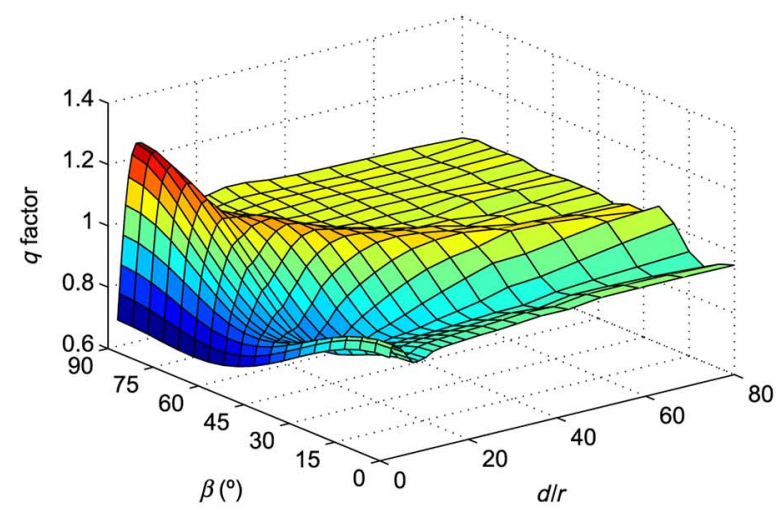

(a)

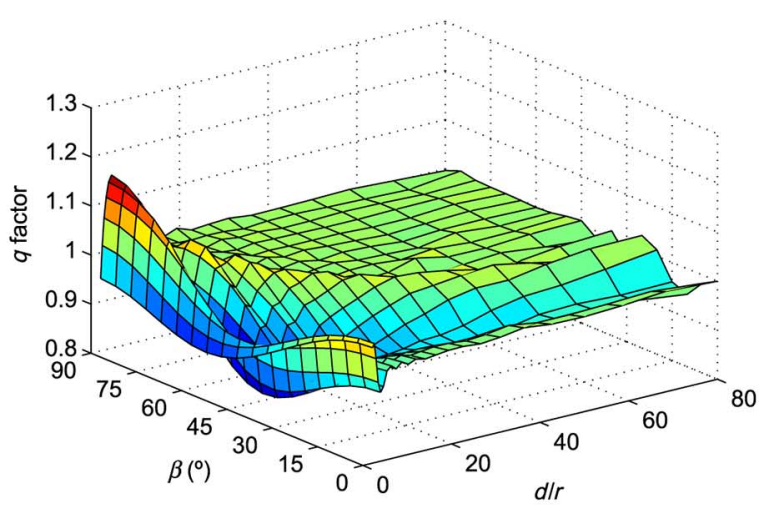

(b)

Fig. 5. $L 2, G 2$ : evolution of the $q$ factor for different values of $d / r$ and $\beta$. (a) GC. (b) PC (IW: $H_{s}=1 \mathrm{~m} ; T_{p}=7 \mathrm{~s}$ ).

be explained by the fact that the $q$ factor curve is an asymptotic curve for a constant $\beta$ and variable $d / r$ ratios [as can be observed in Figs. 2(a) and 3]. The evolution of each of the $q$ factor curves in Figs. 4-7 are smoother or more oscillatory, depending on the interactions between the devices.

\section{DISCUSSION}

Different control strategies affect interactions between the devices in a wave farm in different ways. However, despite different $q$ factor variations with $d / r$ for different control strategies, radiation effects become negligible for larger device separations, so $q \rightarrow 1$ for all control strategies as $d / r$ increases. Figs. 2(a) and 3 clearly illustrate this behavior.

The most significant issue is that the optimal $d / r$ values for the GC and PC strategies are quite different, especially for $T_{p}>$ $T_{r}$. However, the optimal wave farm orientation is not directly influenced by the control strategy applied to the WECs. The influence of wave farm orientation depends on the configuration used for the arrays (i.e., linear, triangular or square), with the control strategy having only a minor effect.

As the number of devices increases and the configuration changes, the interaction between the devices is more complex and the optimal $d / r$ values are generally greater than the values for the two-body linear array case. Fig. 8 illustrates a comparison between the optimal array layouts for the four configurations studied, when PC and GC are applied to the WEC 


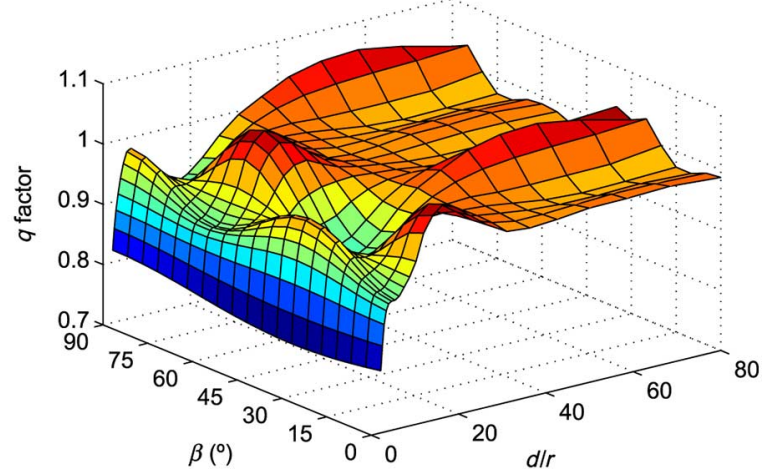

(a)

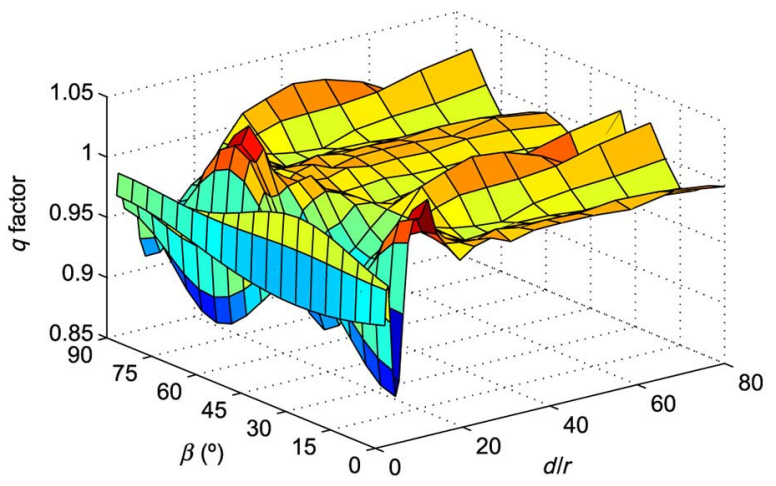

(b)

Fig. 6. $L 3, G 2$ : evolution of the $q$ factor for different values of $d / r$ and $\beta$. (a) GC. (b) PC (IW: $H_{s}=1 \mathrm{~m} ; T_{p}=7 \mathrm{~s}$ ).

farm (G2; IW: $\left.H_{s}=1 \mathrm{~m} ; T_{p}=7 \mathrm{~s}\right)$. Note the significant difference for the optimal separating distances, for the PC- or the GC-controlled cases.

To highlight the impact of not including the control strategy at the layout optimization stage, we design optimal array layouts assuming no control, subsequently apply GC, and compare with the case where the optimal array layout is designed assuming GC is in place (the "control aware" case). The energy absorbed by the arrays is denoted by $E_{\mathrm{nc}}$ and $E_{\mathrm{gc}}$ for the two cases, respectively. Fig. 9 shows the ratio $E_{\mathrm{gc}} / E_{\mathrm{nc}}$ for the four layout configurations. Clearly, the energy capture in the "control aware" case is superior to the "control ignorant" case $\left(E_{\mathrm{gc}} / E_{\mathrm{nc}} \geq 1\right.$ in all cases), with an improvement of up to $40 \%$ in some cases. For linear arrays, in particular, the average benefit of control aware layout design (across all $T_{p}$ ) is over 19\% and $16 \%$, for L1 and L2 layouts, respectively. We note that such performance improvements are significantly greater than any that can be obtained by improved control design ([26] reports an improvement of $8.2 \%$ using model predictive control in comparison to a simple controller), or extension of independent to GC ([16] reports an average improvement of $10-20 \%$ for GC over IC).

In wave farm deployment, the study of the control-informed optimal array layout should consider the mean wave direction and the statistical sea states occurrence of a specific site. Then, the best layout can be defined after running simulations for a different set of wave parameters, e.g., significant wave height and peak period.

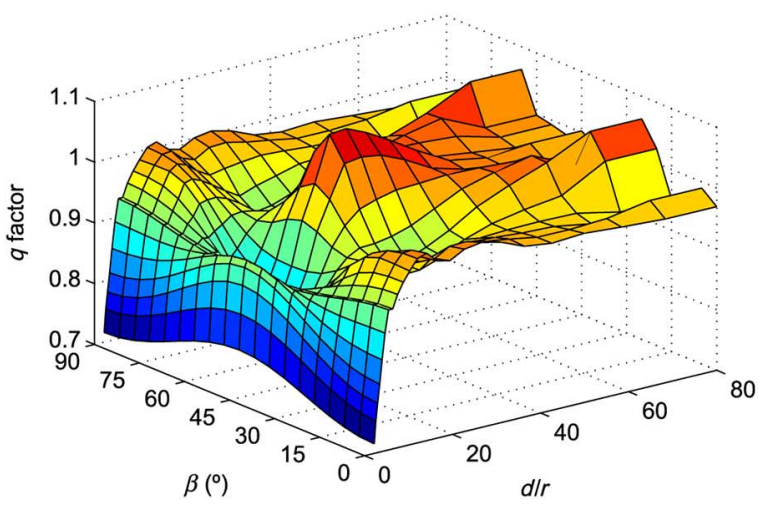

(a)

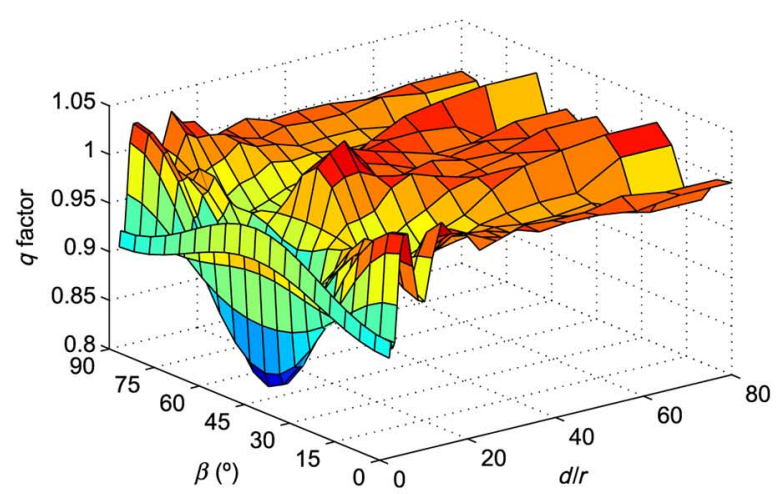

(b)

Fig. 7. $L 4, G 2$ : evolution of the $q$ factor for different values of $d / r$ and $\beta$. (a) GC. (b) PC (IW: $H_{s}=1 \mathrm{~m} ; T_{p}=7 \mathrm{~s}$ ).

L1

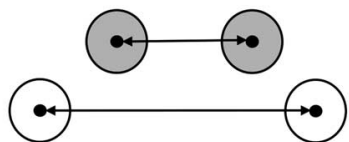

L2
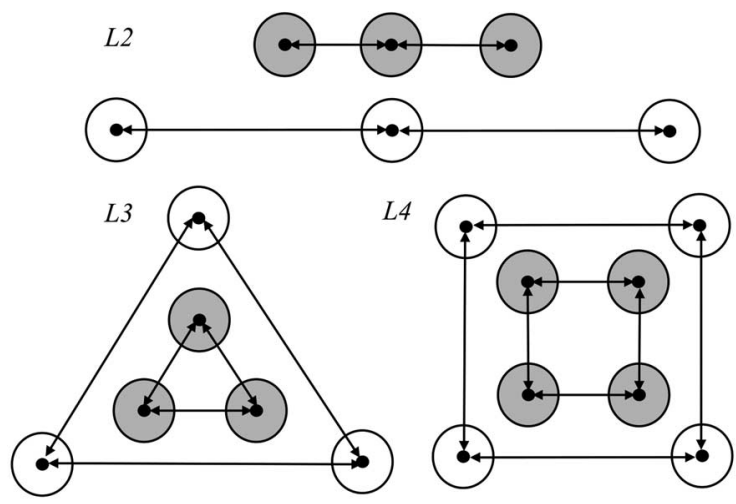

Fig. 8. Top view of optimal arrays configurations for PC (shaded) and GC (unshaded). (a) L1 (scale 1:100). (b) L2 (scale 1:100). (c) L3 (scale 1:200). (d) L4 (scale 1:400).

\section{CONCLUSION}

Consideration of the control scheme is not the only deciding factor in optimal WEC array layout. Other factors may have high priority, including mooring and electrical infrastructure configurations, which have major economic impact. However, the particular control strategy employed clearly does have a causal effect on optimal array separating distances and needs to be considered, together with these other design factors. 


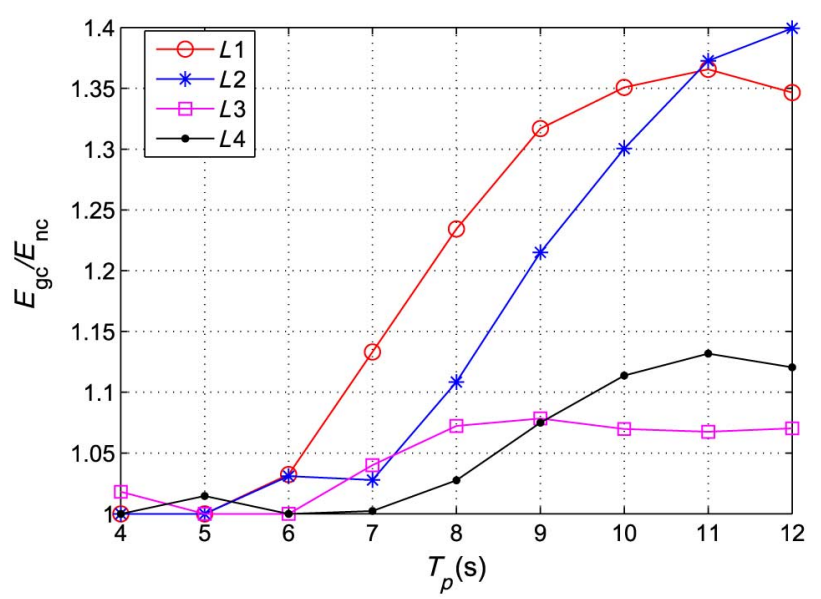

Fig. 9. Ratio between $E_{g c}$ and $E_{n c}$ (IW: $H_{s}=1 \mathrm{~m}$ ).

It is well known that control schemes play an important role in the performance improvement of WECs. However, this paper demonstrates that it is vital to consider the control system to be employed at the array layout stage if the full economic potential of a wave farm is to be realized. In particular, it is demonstrated that the performance improvement from optimal control-informed layout can be up to $40 \%$, which is very significant, considering the current struggle for wave energy to demonstrate economic viability.

\section{REFERENCES}

[1] K. Budal, "Theory for absorption of wave power by a system of interacting bodies," J. Ship Res., vol. 21, no. 4, pp. 248-253, 1977.

[2] Z. Gao and T. Moan, "Mooring system analysis of multiple wave energy converters in a farm configuration," in Proc. 8th Eur. Wave Tidal Energy Conf., Uppsala, Sweden, 2009, pp. 509-518.

[3] F. Sharkey, E. Bannon, M. Conlon, and K. Gaughan, "Maximising value of electrical networks for wave energy converter arrays," Int. J. Mar. Energy, vol. 1, pp. 55-69, 2013.

[4] D. V. Evans, "Some theoretical aspects of threedimensional wave-energy absorbers," in Proc. 1st Symp. Wave Energy Util., Gothenburg, Sweden, 1979, pp. 77-113.

[5] J. Falnes, "Radiation impedance matrix and optimum power absorption for interacting oscillators in surface waves," Appl. Ocean Res., vol. 2, no. 2, pp. 75-80, 1980.

[6] G. P. Thomas and D. V. Evans, "Arrays of three dimensional wave-energy absorbers," J. Fluid Mech., vol. 108, no. 2, pp. 67-88, 1981.

[7] P. McIver and D. V. Evans, "Approximation of wave forces on cylinder arrays," Appl. Ocean Res., vol. 6, no. 2, pp. 101-107, 1984

[8] S. A. Mavrakos and P. McIver, "Comparison of methods for computing hydrodynamic characteristics of arrays of wave power devices," Appl. Ocean Res., vol. 19, no. 5-6, pp. 283-291, 1997.

[9] H. A. Wolgamot, P. H. Taylor, and R. E. Taylor, "The interaction factor and directionality in wave energy arrays," Ocean Eng., vol. 47, pp. 65-73, 2012.

[10] A. D. de Andrés, R. Guanche, L. Meneses, C. Vidal, and I. J. Losada, "Factors that influence array layout on wave energy farms," Ocean Eng., vol. 82, pp. 32-41, 2014.

[11] J. Engström, M. Eriksson, M. Göteman, J. Isberg, and M. Leijon, "Performance of large arrays of point absorbing direct-driven wave energy converters," J. Appl. Phys., vol. 114, no. 204502, 2013.

[12] B. F. M. Child and V. Venugopal, "Optimal configurations of wave energy device arrays," Ocean Eng., vol. 37, no. 16, pp. 1402-1417, 2010.

[13] C. Fitzgerald and G. Thomas, "A preliminary study on the optimal formation of an array of wave power devices," in Proc. 7th Eur. Wave Tidal Energy Conf., Porto, Portugal, 2007.

[14] M. Folley and T. J. T. Whittaker, "The effect of sub-optimal control and the spectral wave climate on the performance of wave energy converter arrays," Appl. Ocean Res., vol. 31, no. 4, pp. 260-266, 2009.
[15] J. Cruz, R. Sykes, P. Siddorn, and R. E. Taylor, "Optimal configurations of wave energy device arrays," IET Renewable Power Gener, vol. 4, no. 6 , pp. 488-497, 2010.

[16] G. Bacelli, P. Balitsky, and J. V. Ringwood, "Coordinated control of arrays of wave energy devices-Benefits over independent control," IEEE Trans. Sustain. Energy, vol. 4, no. 4, pp. 1091-1099, Oct. 2013.

[17] G. Li and M. R. Belmont, "Model predictive control of sea wave energy converters. Part II: The case of an array of devices," Renewable Energy, vol. 68 , pp. 540-549, 2014

[18] A. F. O. Falcão, "Modelling and control of oscillating-body wave energy converters with hydraulic power take-off and gas accumulator," Ocean Eng., vol. 34, pp. 2021-2032, 2007.

[19] J. Falnes, Ocean Waves and Oscillating Systems: Linear Interaction Including Wave-Energy Extraction. Cambridge, U.K.: Cambridge Univ. Press, 2002

[20] P. Balitsky, G. Bacelli, and J. V. Ringwood, "Control-influenced layout optimization of arrays of wave energy converters," in Proc. ASME 33rd Int. Conf. Offshore Mech. Arctic Eng., San Francisco, CA, USA, 2014.

[21] W. E. Cummins, "The impulse response function and ship motions," Schiffstechnik, vol. 47, no. 9, pp. 101-109, 1962.

[22] WAMIT User Manual Versions 6.4, 6.4PC and 6.3S, 6.3S-PC, WAMIT, Inc., Chestnut Hill, MA, USA, 1998-2006.

[23] G. M. Terra, W. J. van de Berg, and L. R. M. Maas, "Experimental verification of Lorentz' linearization procedure for quadratic function," Fluid Dyn. Res., vol. 36, pp. 175-188, 1997.

[24] G. Bacelli, J. V. Ringwood, and J.-C. Gilloteaux, "A control system for a self-reacting point absorber wave energy converter subject to constraints," in Proc. IFAC World Congr., Milan, Italy, 2011, pp. 11 387-11 392.

[25] A. Babarit, "Impact of long separating distances on the energy production of two interacting wave energy converters," Ocean Eng., vol. 37, no. 8-9, pp. 718-729, 2010.

[26] F. Fusco and J. V. Ringwood, "A simple and effective real-time controller for wave energy converters," IEEE Trans. Sustain. Energy, vol. 4, no. 1, pp. 21-30, Jan. 2013.

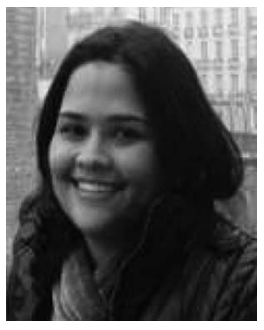

Paula B. Garcia-Rosa (S'13-M'13) received the B.Sc. degree in electronic engineering from the State University of Rio de Janeiro, Rio de Janeiro, Brazil, in 2004, and the M.Sc. and D.Sc. degrees in electrical engineering and ocean engineering, respectively, from the COPPE/Federal University of Rio de Janeiro, Rio de Janeiro, Brazil, in 2008 and 2013, respectively.

She is currently a Postdoctoral Researcher with the Centre for Ocean Energy Research, Maynooth University, Maynooth, Ireland. Her research interests include automation and control of industrial processes, renewable energy technologies and modeling, and optimization and control of ocean energy systems.

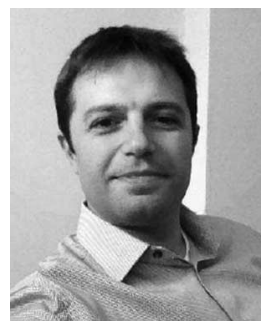

Giorgio Bacelli received the Laurea Magistrale in electronic engineering from the Università Politecnica delle Marche, Ancona, Italy, in 2006, and the Ph.D. degree in electronic engineering from the Centre for Ocean Energy Research, Maynooth University, Maynooth, Ireland, in 2014.

He is currently with Sandia National Laboratories, Albuquerque, NM, USA, working on the design and implementation of control strategies for wave energy converters.

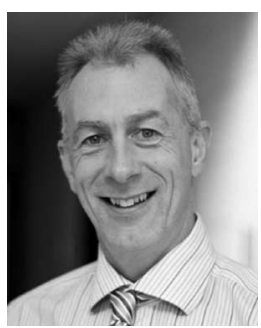

John V. Ringwood (M'87-SM'97) received the Diploma degree in electrical engineering from Dublin Institute of Technology, Dublin, Ireland, in 1981, and the Ph.D. degree in control of steel mills from Strathclyde University, Glasgow, U.K., in 1985.

$\mathrm{He}$ is currently a Professor of Electronic Engineering with Maynooth University, Maynooth, Ireland. $\mathrm{He}$ was the Head of the Electronic Engineering Department, Maynooth University from 2000 to 2006. His research interests include time series modeling, wave energy, control of plasma processes, and biomedical engineering.

Dr. Ringwood is a Chartered Engineer and a Fellow of the Institution of Engineers of Ireland. 